\title{
INVETERATE MIGRAINE TREATMENT USING VIBRATION- A NON- INVASIVE METHOD
}

\author{
S. Shobha Christila ${ }^{1}$, Kanmani. $M^{2}$, Ramya.G ${ }^{3}$, Sathya. $M^{4}$ \\ ${ }^{1}$ Assistant Professor(OG), Department of Biomedical Engineering, Sri Ramakrishna Engineering College, \\ Coimbatore, India \\ ${ }^{2,3,4}$ Project Students, Department of Biomedical Engineering, Sri Ramakrishna Engineering College, Coimbatore, \\ India \\ kanmani.makeswaran@gmail.com
}

\begin{abstract}
Migraine is a neurological syndrome which causes moderate to severe head ache. The migraine headache is associated with nausea, vomiting, photophobia, pulsating sensationmostly at one side of the head and visual disturbances. Most of the migraines are treated and taken care only at the postdromal stage. Acute migraines are treated with the drugs which causes some gastro intestinal problems, chest pain, reduce in the blood pressure, dizziness. The other method for migraine treatment includes neurostimulation and nerve decompression.This project embraces the use of non-invasive method using a vibration where it is placed at the acupressure points. These points when used in a right manner can relieve Headaches and migraine. The vibration is created by a set of electronic components and arduino where the vibration frequency can be changed manually as per the wish of the patient's comfort. These readings of frequencies used with respect to the digital samples can be monitored by the external SD card where it can be further verified by the doctor on special cases.
\end{abstract}

KeyWords: NerveDecompression, Dromal stage, Neurostimulation

\section{INTRODUCTION}

Migraine is a severe disabling headache, usually affecting only one side of the head. It is a neurological syndrome and it is usually associated with the combination of two or more symptoms. Here it is the headache along with nausea, vomiting, photophobia and visual disturbance. The diagnosis of this syndrome is kind of a very difficult task. But the treatment for this migraine is carried out such as drug therapy like triptans, asprin etc. and invasive treatment such asgamma knife, nerve decompression and neurostimulation.

Even though these were the entire effective one when it comes to the treatment, the drug therapy lead to the toxicity, ulcers, parathesia and even the surgery proved to be a viable option it could be used only to selected patients depending upon their condition. Hence non-invasive methods like using magnets and upper cervical Chiropractic were used. In order to provide a little more comfort to the patients and to satisfy their needs economically, the vibration treatment was followed were the device is handheld and could be used with simple instructions. This method produces vibration which could be placed at the acupressure points and then the vibrations provide a kind of stimulation and hence the migraine can be treated accordingly.

\section{MIGRAINE}

Migraine is a neurological disorder which is recurrent moderate to heavy headaches.it affects one side of the head or half of the head and its duration from 2 to 72 hours. Globally $15 \%$ of people affected by migraine. Migraine causes due to both environmental and genetic factors. High level of neurotransmitter serotonin (5-hydroxytryptamine) and increased excitability of the cerebral cortex and abnormal control of pain neurons in the trigeminal nucleus of the brainstem. The major symptoms are aura, nausea, vomiting, sensitivity to light, sound and smell. Migraine has four phases which are prodrome, aura, pain or headache, postdrome. The prodromal phase is the primary phase before the start of aura. This stage from 2 hours to 2 days.this phase symptoms are altered mood, irritated, depression, euphoria, fatigue, craving for certain foods, stiff muscles, constipation or diarrhoea and sensation to smell. The second phase is aura which is transient focal neurological phenomenon. It occurs before or during the pain. Aura appears and last within one hour. During these stage symptoms are visual, sensory and motor effects.

Visual disturbances consist of scintillating scotoma which is partial alteration in the field of vision and hemianopia which is loss of part of their vision. The other symptoms are language disturbances, word spinning, and motor problems. The third phase headache is unilateral, throbbing, and moderate to severe in intensity and the pain may be bilateral and neck pain. Bilateral pain is common in the migraine without aura and neck or top of the head pain is primarily occurs. The pain duration ranges from 4 to 72 hours in adults and less than one hour in children and the pain is accompanied by nausea. In $90 \%$ of people, vomiting sensation, fatigue, irritability, light-headedness, confusion, blurred vision, pallor and sweating. Aura occurs without a subsequent headache which is known as acephalgic 
migraine or silent migraine. The final phase postdrome is the effects of migraine may persist for some days after the main headache has to be ended. Major problems are sore feeling in the area where the migraine occurs, impaired thinking for few days after the headache, patient may feel tired or hung over and euphoric after an attack but this duration can vary from one to another. Migraines are divided into seven subclasses which are migraine without aura or common migraine, familial hemiplegic migraine, sporadic migraine which are migraine with aura. Basilar type migraine where headache and aura accompanied by difficulty of speaking, word spinning, ringing in ears and brainstem related symptoms.Retinal migraine causes visual disturbances or temporary blindness in one eye. Chronic migraine is a complication of migraine and abdominal migraine with precursors includes cyclical vomiting syndrome and benign paroxysmal vertigo of childhood.

\section{EARLIER METHODS TO TREAT MIGRAINE}

Most widely used invasive methods for the treatment of chronic migraine headache are as follows: Nerve decompression is one of the invasive methods to treat the migraine head ache. In this, the pressures created by the pinched or entrapped nerves are decompressed to cure the migraine headache. The nerves involved are supraorbital nerve, supratrochealar nerve and greater occipital nerve.Neurostimulation is one of the minimal invasive methods for the treatment of the chronic migraine headache. In this surgical procedure, light electrical current to excite the nerves which delivers the pain signals to the brain.

Magnetic stimulation is one way to treat the chronic migraine headache in non-invasive manner. In this, magnetic stimulation is applied to the back of the head in which magnetic field that induce electric currents. The most widely used device for magnetic stimulation is eNeuraElectrical stimulation is another way for the treatment of migraine headache in non-invasive manner. The most widely used device for electrical stimulation is Cefaly. Chiropractic therapy is also other method or therapy for the treatment of chronic migraine headache. It involves the process of stretching, moving and manipulating the spine.

\section{INVETERATE MIGRAINE TREATMENT USING VIBRATION}

Vibrations are used on a wide range of applications. It is used in the medical field as a therapeutic purpose. Vibration therapy can be used for the whole body and on the localized areas of your body. During this type of localized area therapeutic purpose the practitioner uses a hand-held device which can be placed at different places and thus therapy is followed. This vibration therapy works on the principle of frequency, which could be manually operated by the individual according to the individual's need. The direction of use and the intensity of the vibration used is the one which makes it effective.
Acupressure is the one which insists on the energy flow through certain points in the body called the meridians. Any physical pressure can be given with the help of hand, elbow or any devices to these meridian points which is said to clear the blockages if any. The blockages are the ones which create the pain. This inveterate migraine treatment uses the acupressure points such as Third eye point, Wind Mansion, Gates of Consciousness, Heavenly Pillar, and Above Tears where if a pressure or vibrational energy given to these points relieves the pain.

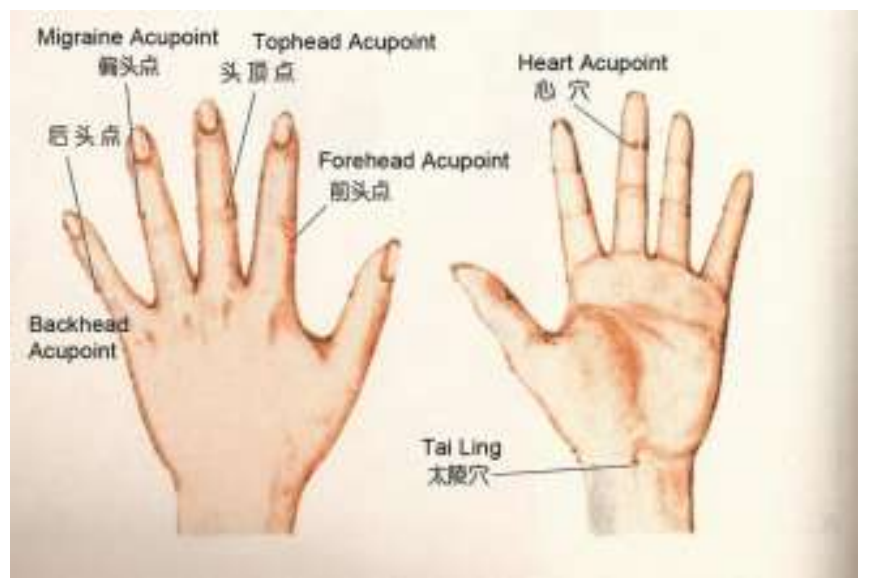

Fig 1. Accupressure points in hand

The nerve serves for two causes. One for the motor neurons, another for the sensory neurons. Here the device stimulates vibrational energy thereby stimulating the sensory fibres of the nerves. Sensory neurons are the ones which can detect the changes in the environment thus giving the required information to the brain and spinal cord for further processing. When mechanoreceptors fail to function properly then there arise the disorders such as neuropathic pain which results in the damaged sensory nerve. To serve this purpose of sensation to pain the vibrational energy is given to the acupressure regions of specific site.
Arduino Pin Mapping

\begin{tabular}{|c|c|c|c|}
\hline & & 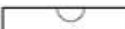 & \\
\hline & (RESET) PC6 & 1 & 8 PC5 (ADC5/SCL) \\
\hline digital pin $0(\mathrm{RX})$ & (RXD) PDO & 2 & 7 PC4 (ADC4/SDA \\
\hline digital pin 1 (TX) & (TXD) PD1 & 3 & 6 PC3 (ADC3) \\
\hline digital pin 2 & (INTO) PD2 & 4 & 5 PC2 (ADC2) \\
\hline digital pin 3 & (INT1) PD3 & 5 & $4 P^{-1} 1$ ( $A D C 1$ ) \\
\hline digital pin 4 & (XCK/TO) PD4 & 6 & $3 . P C O(A D C D)$ \\
\hline & $\operatorname{vcc}$ & 7 & 2 G GND \\
\hline & GND & 8 & 1 AREF \\
\hline & (XTAL1/TOSC1) PB6 & 9 & OPaVcc \\
\hline & (XTAL2/TOSC2) PB7 & 10 & 9 PB5 (SCK) \\
\hline digital pin 5 & (T1) PD5 & 11 & 8 P PB4 (MISO) \\
\hline digital pin 6 & (AINO) PD6 & 12 & 7 P PB3 (MOSI/OC2) \\
\hline digital pin 7 & (AIN1) PD7 & 13 & 6 PB2 (SS/OC1B) \\
\hline digital pin 8 & (ICP1) PBO L & 14 & 5 P PB1 (OC1A) \\
\hline
\end{tabular}

Fig 2. Pin configuration of Atmega 328 www.arduino.ce

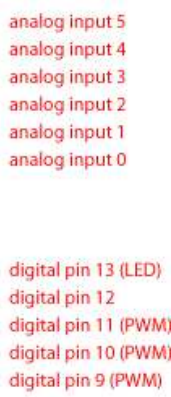

digital pin 9 (PWM) 


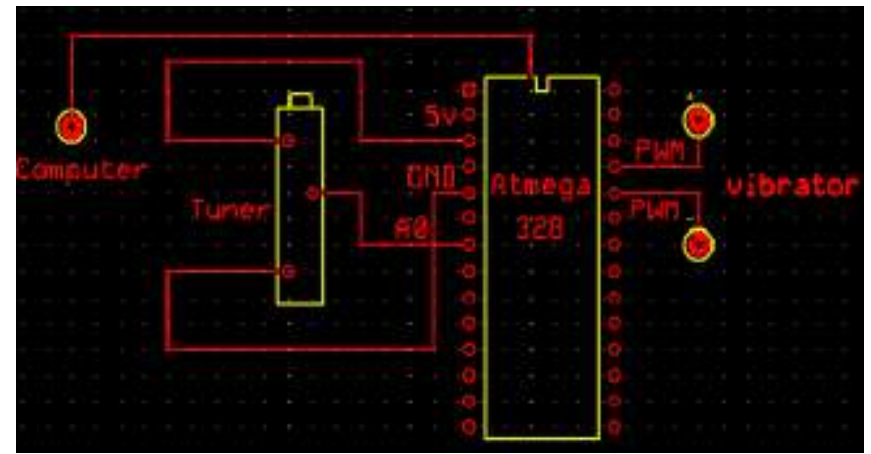

Fig 3. PCB design

\section{IV.1.WORKING}

The device comprises a mould, potentiometer; vibratory motor which is run by the programmed arduino. Thearduino which works as a microcontroller here is powered and controlled by the computer device. The vibratory motor is connected to the control unit and this control unit is controlled by the power circuit or the computer device. The mould which is in the form of the band touching the back of the ear or the temporal region. The vibratory motor which is embedded within the housing transmits the vibrational energy to the outer walls of the housing. This in turn vibrates the mould which is in contact with the back of the ear (skin). The vibration is given for a stipulated amount of time where the frequency changes are manual according to the subject's requirement.

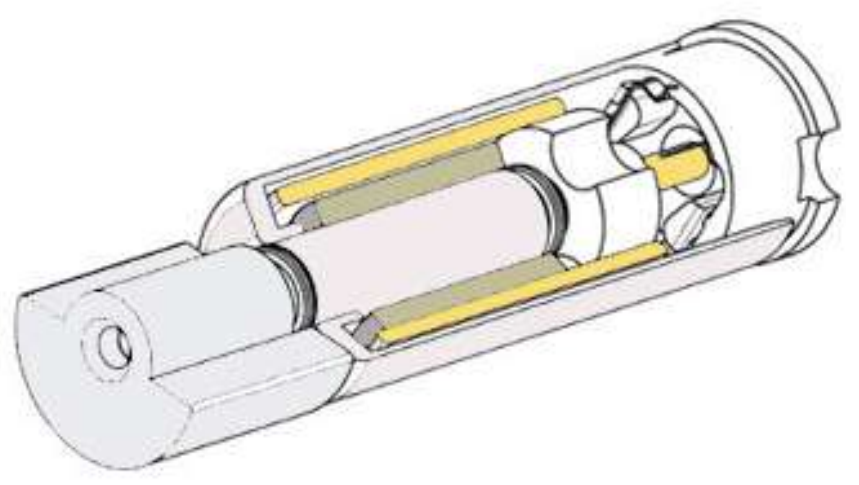

Fig 4. Vibratory Motor

Once when the analog signals are given as power to controlling device where the maximum power will be about $5 \mathrm{~V}$ to $7 \mathrm{~V}$. Then this anolog signals are then converted to the digital samples where the output is controlled by the potentiometer aka known as variable resistance. When the digital samples are acquired, these are converted to the requirement of the problem. The migraine treatment is done at a frequency of about $0-150 \mathrm{~Hz}$. Hence the digital samples of about $0-1023$ are converted between $0-150 \mathrm{~Hz}$ frequencies where these outputs are connected to the potentiometer. The potentiometer then changes or modifies the speed of the rotation of the vibratory motor which is a manual process.

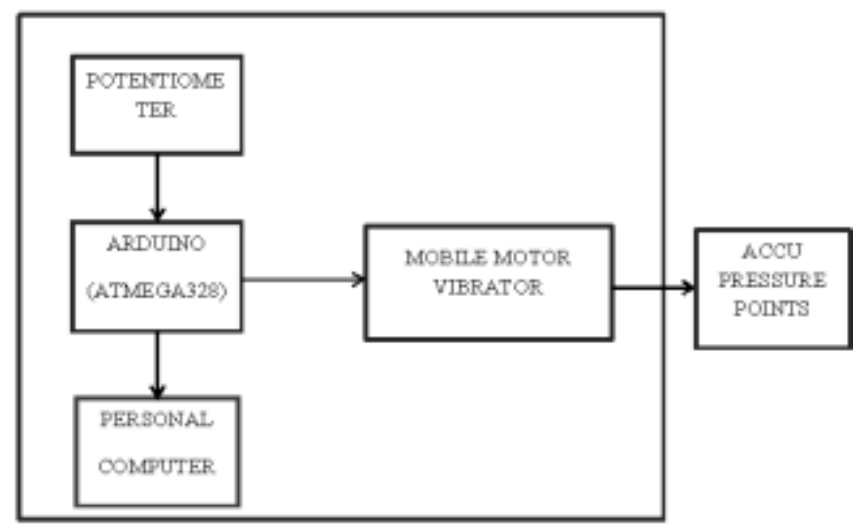

Fig 5. Schematic Diagram

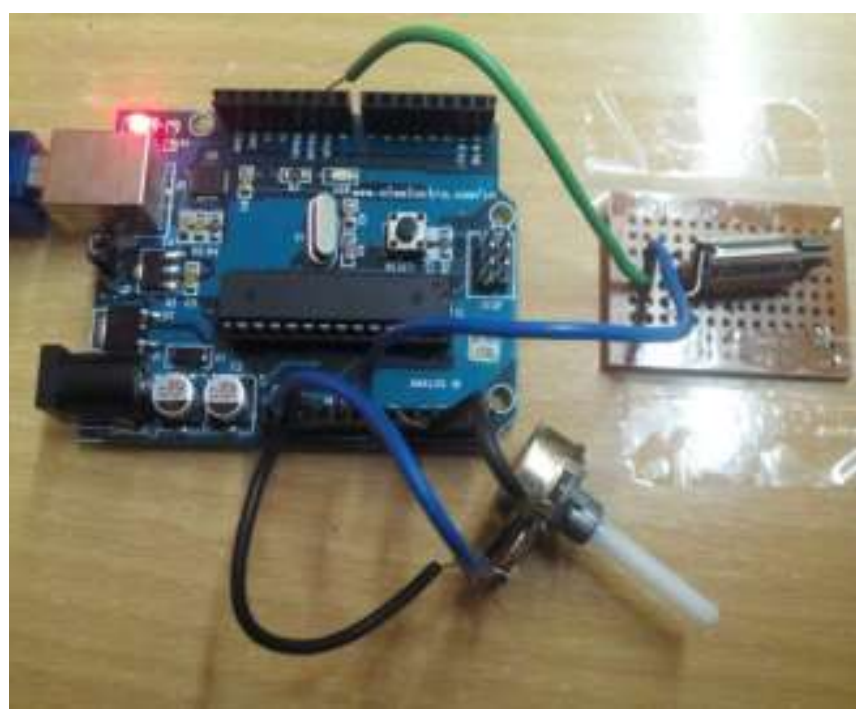

Fig 6. Circuit Diagram

\section{V.CONCLUSION}

Thus this treatment of inveterate migraine using vibration serves to be a drug free and better tolerant and a noninvasive method. The mould is designed in such a way that the subjects are comfortable with the wearing of the mould and the cost effectiveness of the device. It is radiation less and thus provides an ease of painless treatment. The acupressure region which is followed all through the ancient days is brought back with the combination of new technologies. The treatment can also be looked forward for the less time period for the recovery.

\section{RESULTS}

The future work will be based on the case study where a stipulated amount of subjects can be examined for the treatment and the response of each and every one for the time of recovery is noted.

\section{REFERENCES}

[1] Piero Barbanti1, Licia Grazzi2, Gabriella Egeo1, Anna Maria Padovan3, Eric Liebler4 and Gennaro Bussone2 "Non-invasive vagus nerve stimulation for acute treatment of high-frequency and chronic migraine"- 
Barbanti et al. The Journal of Headache and Pain (2015) 16:61.

[2] Eneura,INC.,Sunnyvale,CA(US).“Transcranial magnetic stimulation device with body proximity sensors for the treatment of migraine headache"-United States Patent Application Publications, 2014(14/315,994).

[3] Lipton RB,Stewart WF .Acute migraine therapy"2012;39:S20-S26.

[4] https://www.google.co.in/search?q=pin+diagram+for+a tmega328\&sa=G\&tbm=isch\&imgil

[5] Brønfort G, Nilsson N, Haas M, Evans RL, Goldsmith $\mathrm{CH}$, Assendelft WJJ, Bouter LM. "Non-invasive physical treatments for chronic/recurrent headache (Review)" -The Cochrane Library2004, Issue 3.

[6] "Non-invasive vagus nerve stimulation foracute treatment of high-frequency andchronic migraine: an open-label study" .Piero Barbanti1, Licia Grazzi2, Gabriella Egeo1, Anna Maria Padovan3, Eric Liebler4 and Gennaro. Barbanti et al. The Journal of Headache and Pain (2015) 16:61 DOI 10.1186/s10194-015-0542-4 